\title{
RF Performance and Modeling of Si/SiGe Resonant Interband Tunneling Diodes
}

\author{
Niu Jin, Member, IEEE, Sung-Yong Chung, Student Member, IEEE, Ronghua Yu, \\ Sandro J. Di Giacomo, Member, IEEE, Paul R. Berger, Senior Member, IEEE, and \\ Phillip E. Thompson, Senior Member, IEEE
}

\begin{abstract}
The RF performance of two different Si-based resonant interband tunneling diodes (RITD) grown by low-temperature molecular beam epitaxy (LT-MBE) were studied. An RITD with an active region of $B \delta$-doping plane $/ 2 \mathrm{~nm}$ i-Si $i_{0.5} \mathrm{Ge}_{0.5} / 1 \mathrm{~nm}$ $\mathrm{i}$-Si/P $\delta$-doping plane yielded a peak-to-valley current ratio (PVCR) of 1.14, resistive cutoff frequency $\left(f_{r_{0}}\right)$ of $5.6 \mathrm{GHz}$, and a speed index of $23.3 \mathrm{mV} / \mathrm{ps}$ after rapid thermal annealing at $650{ }^{\circ} \mathrm{C}$ for $1 \mathrm{~min}$. To the authors' knowledge, these are the highest reported values for any epitaxially grown Si-based tunnel diode. Another RITD design with an active region of $1 \mathrm{~nm} \mathbf{p +}$ $\mathrm{Si}_{0.6} \mathrm{Ge}_{0.4} / \mathrm{B} \delta$-doping plane/4-nm i- $\mathrm{Si}_{0.6} \mathrm{Ge}_{0.4} / 2 \mathrm{~nm}$ i-Si/P $\delta$-doping plane and annealed at $825{ }^{\circ} \mathrm{C}$ for $1 \mathrm{~min}$ had a PVCR of 2.9, an $f_{r 0}$ of $0.4 \mathrm{GHz}$, and a speed index of $0.2 \mathrm{mV} / \mathrm{ps}$. A small signal model was established to fit the measured $S_{11}$ data for both device designs. Approaches to increase $f_{r 0}$ are suggested based on the comparison between these two diodes. The two devices exhibit substantially different junction capacitance/bias relationships, which may suggest the confined states in the $\delta$-doped quantum well are preserved after annealing at lower temperatures but are reduced at higher temperature annealing. A comprehensive dc/RF semi-physical model was developed and implemented in Agilent advanced design system (ADS) software. Instabilities in the negative differential resistance (NDR) region during dc measurements were then simulated.
\end{abstract}

Index Terms-Modeling, molecular beam epitaxial (MBE) growth, $\mathrm{RF}$ performance, $\mathrm{Si} / \mathrm{SiGe}$ heterojunction, tunnel diodes.

\section{INTRODUCTION}

$\mathbf{S}_{\mathrm{s}}^{\mathrm{n}}$ INCE Si-based resonant interband tunneling diodes (RITD) grown by low-temperature molecular beam epitaxy (LTMBE) were first demonstrated by Rommel et al. [1], numerous studies have been carried out to improve their dc performance [2]-[5]. With recent demonstrations of monolithic integrations of RITDs with RITDs [6], HBTs [7], and CMOS [8], it is of

Manuscript received June 20, 2005. The work done at The Ohio State University was supported by the National Science Foundation under Grants DMR0103248 and ECS-0323657. The work performed at the National Research Laboratories was supported by the Office of Naval Research. The review of this paper was arranged by Editor M. Anwar.

N. Jin was with the Department of Electrical and Computer Engineering, The Ohio State University, Columbus, OH 43210 USA. He is now with the Micro and Nanotechnology Laboratory, University of Illinois, Urbana, IL 61801 USA.

S.-Y. Chung, and S. J. Di Giacomo are with the Department of Electrical and Computer Engineering, The Ohio State University, Columbus, OH 43210 USA.

$\mathrm{R}$. Yu is with the Department of Physics, The Ohio State University, Columbus, OH 43210 USA.

P. R. Berger is with the Department of Electrical and Computer Engineering and the Department of Physics, The Ohio State University, Columbus, OH 43210 USA (e-mail: pberger@ieee.org).

P. E. Thompson is with the Naval Research Laboratory, Washington, DC 20375 USA.

Digital Object Identifier 10.1109/TED.2005.856183

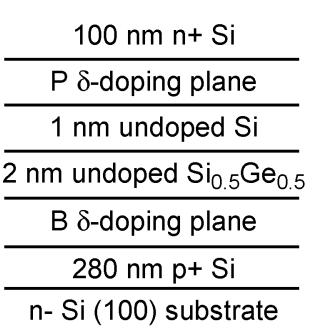

(a)

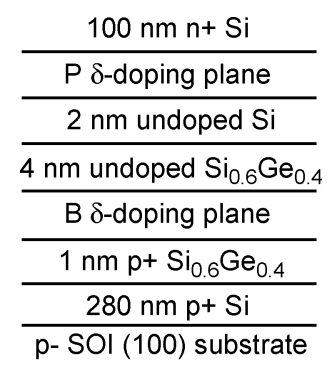

(b)
Fig. 1. Schematic of the RITD layer structures used in this paper. (a) Structure A is an RITD with a 3-nm-thick spacer. (b) Structure B is an RITD with a 6-nm-thick spacer.

prime interest to characterize their microwave performance, especially their resistive cutoff frequency $f_{r 0}$ and speed index $s$ which is defined as the quotient of peak current over capacitance, for high-speed circuit applications. Microwave characterization of RITDs facilitates the establishment of an appropriate comprehensive dc/RF model that enables future RITD/transistor circuit development [9].

For comparison, a typical 1960s Si alloyed Esaki diode exhibits a speed index of $1.2 \mathrm{mV} / \mathrm{ps}$ [10]. Recently, Auer et al. reported an epitaxially grown $\mathrm{Si} / \mathrm{SiGe}$ ITD with a speed index of $0.5 \mathrm{mV} / \mathrm{ps}$ and an $f_{r 0}$ estimated as $1.5 \mathrm{GHz}$ [11]. Dashiell et al. reported an epitaxially grown $\mathrm{Si}$ Esaki diode with a speed index of $7.1 \mathrm{mV} / \mathrm{ps}$ and $f_{r 0}$ of $1.6 \mathrm{GHz}$ at $260 \mathrm{~K}$ [12]. In this paper, we studied the RF performance of two RITDs with different spacer configurations and annealing temperatures. The $650{ }^{\circ} \mathrm{C}$ annealed 3-nm-spacer RITD exhibits a resistive cutoff frequency $f_{r 0}$ of $5.6 \mathrm{GHz}$ and a speed index of $23.3 \mathrm{mV} / \mathrm{ps}$ at room temperature, which are both the highest experimental values ever reported for any Si-based tunnel diodes. Based on the comparison of the RF performance and extracted circuit parameters of these two RITDs, several approaches are given to increase the $f_{r 0}$ of Si-based tunnel diodes. A comprehensive $\mathrm{dc} / \mathrm{RF}$ tunnel diode model was developed and implemented in advanced design system (ADS) software (Agilent). DC instabilities during measurement over the negative differential resistance (NDR) region were then simulated.

\section{LAYer Design AND Device FABRICATION}

Fig. 1(a) and (b) shows the schematic of the RITD structures used in this paper. Structure A used a 3-nm-thick intrinsic tunneling spacer with an active region of B $\delta$-doped $\mathrm{Si} / 2 \mathrm{~nm}$ 
i-Si ${ }_{0.5} \mathrm{Ge}_{0.5} / 1 \mathrm{~nm} \mathrm{i}-\mathrm{Si} / \mathrm{P} \delta$-doped $\mathrm{Si}$. It was designed to maximize the current density span, the difference between the peak and valley current densities. Compared to a previously reported structure showing the largest current density span of $76 \mathrm{kA} / \mathrm{cm}^{2}$ [5], the Ge content was increased from $40 \%$ to $50 \%$ to reduce the tunneling barrier height and hence increase the peak current density (PCD). The increased Ge concentration also leads to better momentum mixing and less $\mathrm{B}$ diffusion into the tunneling barrier. The 1-nm SiGe cladding layer typically added under the $\mathrm{B} \delta$-doping layer [4] was removed to ensure the aggregate $\mathrm{SiGe}$ layer thickness did not exceed the total critical thickness. Structure B has a 6-nm-thick intrinsic spacer with an active region of $1-\mathrm{nm} \mathrm{p}+\mathrm{Si}_{0.6} \mathrm{Ge}_{0.4} / \mathrm{B} \delta$-doped $\mathrm{Si} / 4 \mathrm{~nm}$ i-Si $\mathrm{S}_{0.6} \mathrm{Ge}_{0.4} / 2 \mathrm{~nm} \mathrm{i}-\mathrm{Si} / \mathrm{P} \delta$-doped $\mathrm{Si}$, which was developed to maximize the peak-to-valley current ratio (PVCR) [4].

The two structures were grown by LT-MBE using elemental $\mathrm{Si}$ and $\mathrm{Ge}$ in electron-beam sources. The nominal doping level for both $\mathrm{n}^{+}$and $\mathrm{p}^{+}$injector layers are $5 \times 10^{19} \mathrm{~cm}^{-3}$, while both the $\mathrm{B}$ and $\mathrm{P} \delta$-doping sheet concentrations were maintained at $1 \times 10^{14} \mathrm{~cm}^{-2}$. Structure A was grown on a 75-mm P-doped ( $\rho=3-7 \Omega \cdot \mathrm{cm}) \mathrm{Si}(100)$ wafer, while Structure B was grown on a 100-mm B-doped silicon-on-insulator (SOI) (100) wafer.

Prior to device fabrication, portions of the as-grown wafers were rapid thermal annealed using a forming gas ambient $\left(\mathrm{N}_{2} / \mathrm{H}_{2}\right)$ at various temperatures for $1 \mathrm{~min}$. Arrays of diodes were fabricated using standard contact photolithography. Structure B was processed using a conventional double-mesa structure. The first mesa with a $10-\mu \mathrm{m}$ diameter was defined by photoresist and formed by etching through the $\mathrm{p}-\mathrm{n}$ junction using a $\mathrm{HF} / \mathrm{HNO}_{3} / \mathrm{H}_{2} \mathrm{O}(1 / 100 / 100)$ solution. The second mesa was aligned with the first mesa and formed by etching down to the $\mathrm{SiO}_{2}$ layer using the same chemistry. A photosensitive polyimide layer, $800 \mathrm{~nm}$ thick, was then spincoated on the wafer followed by photolithography to open contact windows to both the anode and cathode. After curing the polyimide layer in a $\mathrm{N}_{2}$ ambient, another level of lithography was performed to define the probe pads. Finally, a Ti/Al/Au (15/100/200 nm) multilayer for both ohmic contact and RF probing pads was deposited followed by liftoff. No contact annealing was performed.

The above-mentioned process is not suitable for the fabrication of Structure A due to stability issues caused by the ultrahigh PCD. To make a valid RF measurement, the tunnel diode should be stable when it is biased in its NDR region. The stability criteria [13] is

$$
R<\frac{\left(V_{v}-V_{p}\right)}{\left(I_{p}-I_{v}\right)}
$$

and

$$
\frac{L}{R}<C\left|R_{n}\right|
$$

where $R$ and $L$ are the series resistance and inductance, respectively. $C$ is the junction capacitance and $R_{n}$ is the differential resistance $\mathrm{dV} / \mathrm{dI}$. Oscillations will take place in the NDR region if either of these two criteria are not satisfied. Therefore, Structure A with an ultralarge current density span should have a very small diode size and small series

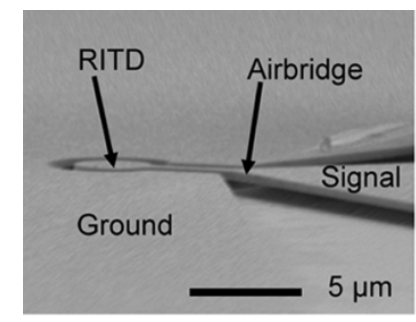

(a)

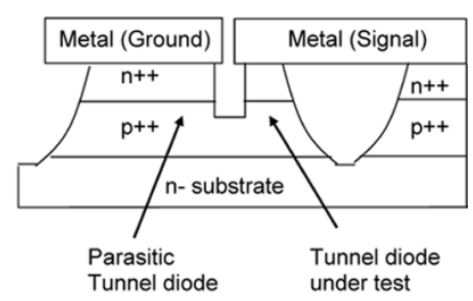

(b)
Fig. 2. (a) SEM photomicrograph of a fabricated RITD (Structure A). The airbridge is clearly visible. (b) Schematic view of the device structure.

resistance to satisfy the first inequality (1). A 1-metal fabrication process [11] and self-aligned $\mathrm{Ni}$ silicidation process was employed to fabricate Structure A. The first lithography step defines both the anode and cathode contact, as well as the probe pads, followed by deposition and lift-off of $\mathrm{Ni} / \mathrm{Cr} / \mathrm{Au}$ $(6 / 100 / 200 \mathrm{~nm})$ using electron beam evaporation. After lift-off, the sample was annealed at $420{ }^{\circ} \mathrm{C}$ for $5 \mathrm{~min}$ to form the $\mathrm{Ni}$ silicide. $\mathrm{Cr}$ prevents the interdiffusion of $\mathrm{Au}$ into the silicide. $\mathrm{KOH} / \mathrm{IPA} / \mathrm{H}_{2} \mathrm{O}(76 / 50 / 140)$ solution was used to etch through the tunnel junction. The mesas were oriented $45^{\circ}$ to the $\langle 110\rangle$ direction to form a vertical sidewall. The second lithography step defined a photoresist etching mask that protects the RITD mesa but defines the isolation area. The second etching step uses an $\mathrm{HF} / \mathrm{HNO}_{3} / \mathrm{H}_{2} \mathrm{O}(1 / 100 / 100)$ solution to etch down to the $\mathrm{n}$ - substrate. This etching step effectively isolates the active mesa from the epi-layer under the signal pad. Fig. 2(a) shows a scanning electron microscopy (SEM) photomicrograph of a representative device. The airbridge formed by the large undercut is clearly visible. Fig. 2(b) illustrates the device structure from a cross-sectional view along the finger. Note that the serially connected parasitic tunnel diode under the ground pad will be reverse biased when the device under test (DUT) is forward biased. This junction acts as an extremely small resistance due to its large size $\left(\sim 0.05 \mathrm{~mm}^{2}\right)$ and inherent large junction conductance resulting from the thin tunneling barrier.

The active mesa area of the fabricated RITD is greatly dependent on the amount of undercut created during the second etching step. To estimate the active device area, the PCD of a $5 \times 5 \mu \mathrm{m}$ calibration diode was measured before the second etching step. Since no clear dependence of PCD on junction area was observed, the active device area for Structure A studied in this work can then be estimated by assuming a constant PCD with high accuracy.

\section{DEVICE PERFoRMANCE}

The room temperature dc current-voltage $(I-V)$ characteristics were measured using an Agilent 4156C parameter analyzer. A detailed analysis of each sample processed was performed that tested a large number of devices and representative $I-V$ characteristics recorded. Structure A, annealed at $650{ }^{\circ} \mathrm{C}$ for $1 \mathrm{~min}$, resulted in a PVCR of 1.14 with a peak current of $5.4 \mathrm{~mA}$ ( $I-V$ characteristics shown in Fig. 5). The estimated PCD and junction area are $152 \mathrm{kA} / \mathrm{cm}^{2}$ and $3.55 \mu \mathrm{m}^{2}$, respectively, which is comparable to the performance of a previously reported Si-based RITD annealed at $575^{\circ} \mathrm{C}$ showing a record 


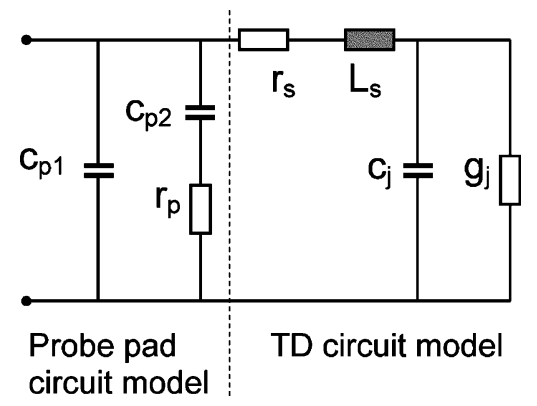

Fig. 3. Small-signal equivalent circuit of the RITDs and the probe pads.

high PCD of $151 \mathrm{kA} / \mathrm{cm}^{2}$ with a PVCR of 2.0 [5]. Structure B, annealed at $825{ }^{\circ} \mathrm{C}$ for $1 \mathrm{~min}$, resulted in a PVCR of 2.9 with PCD of $316 \mathrm{~A} / \mathrm{cm}^{2}$ ( $I-V$ characteristics shown in Fig. 8). Note the 6-nm spacer results in a higher PVCR but much smaller PCD than the 3-nm spacer, due to the exponential decay of current through thicker barriers and enhanced selectivity rules.

Small signal RF performance of representative devices were characterized using an Agilent 8510C network analyzer. The $50 \Omega$ coplanar microwave probes were calibrated by the shortopen-load-through method before measurements of the diodes. $S_{11}$ of both diodes and on-wafer short- and open-pad test structures were measured from $100 \mathrm{MHz}$ to $20 \mathrm{GHz}$ in steps of $0.1 \mathrm{GHz}$. The bias voltage was swept from $0 \mathrm{mV}$ to $0.7 \mathrm{~V}$ in 10 to $50 \mathrm{mV}$ steps. The RF signal delivered to the diodes was estimated to be $10 \mathrm{mV}$ peak-to-peak.

Although the 6-nm-spacer and 3-nm-spacer RITDs were fabricated using different methods and have different structures, their probing pads can be modeled using the same basic equivalent circuit model, shown in Fig. 3. $C_{p 1}$ and $r_{p}$ represent the fringe capacitance and resistive substrate loss, respectively for both structures. $C_{p 2}$ represents the PN junction capacitance for the 3-nm-spacer RITD grown on n- type substrate and $\mathrm{SiO}_{2}$ capacitance for the 6-nm-spacer RITD grown on p- type SOI substrate. The intrinsic RITDs were modeled with a series resistance $\left(r_{s}\right)$, series inductance $\left(L_{s}\right)$, junction capacitance $\left(C_{j}\right)$, and junction conductance $\left(g_{j}\right)$. ADS software was used to fit the measured $S_{11}$ data to the equivalent circuit model. The pad parameters were determined by fitting the equivalent circuit model to measured open- and short-pad test structures. The intrinsic $S_{11}$ data of the measured RITDs was then deembedded from the parasitic effects of the probe pads. The individual circuit parameters of the tunnel diode model were then determined by fitting the equivalent circuit model to the deembedded $S_{11}$ data for each of the RITDs.

Bias dependent $f_{r 0}$ of Structure A annealed at $650^{\circ} \mathrm{C}$ with an estimated active area of $3.55 \mu \mathrm{m}^{2}$ is plotted in the inset of Fig. 4. By biasing the diode at $580 \mathrm{mV}$ (NDR region), the RITD exhibits a $f_{r 0}$ of $5.6 \mathrm{GHz}$, which is nearly four times higher than previously reported values [11], [12]. To the authors' knowledge, this is the highest $f_{r 0}$ ever reported for any epitaxially grown Si-based tunnel diode. The symbols in Fig. 4 represent the measured impedance of the intrinsic diode, which was computed from the deembedded $S_{11}$, as a function of frequency at three representative bias voltages: $500 \mathrm{mV}$ (prepeak region), $580 \mathrm{mV}$ (NDR region) and $700 \mathrm{mV}$ (post-valley region). The solid lines in Fig. 4 are the fitted impedance, which is in very

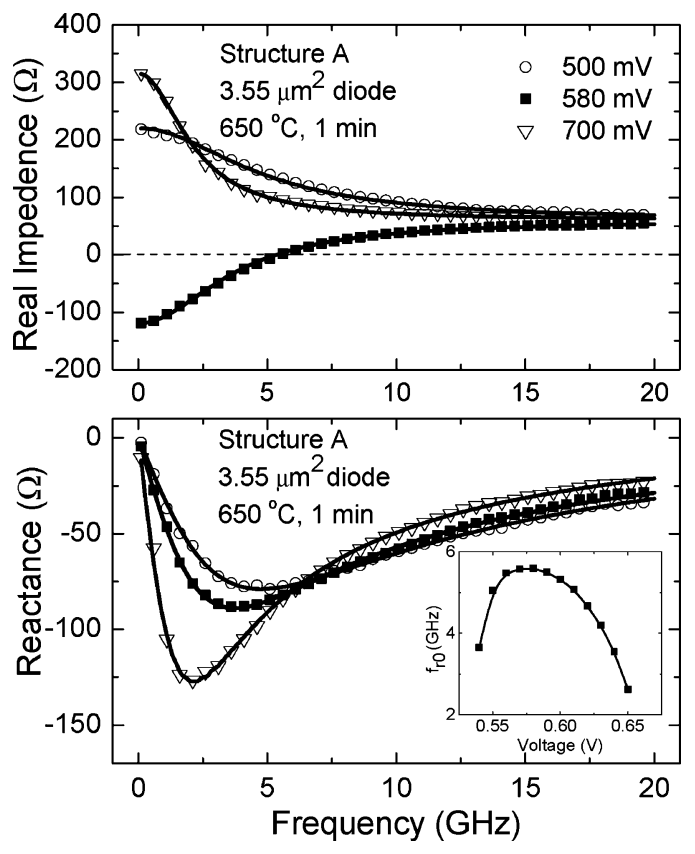

Fig. 4. Comparison of measured impedance (scattered symbols) and modeled impedance (solid lines) at 3 representative bias voltages for Structure A annealed at $650{ }^{\circ} \mathrm{C}$. The real components are plotted in the upper figure and the imaginary components are plotted in the lower figure. The three bias voltages plotted are 500 (prepeak region), 580 (NDR region), and $700 \mathrm{mV}$ (post-valley region). The inset shows $f_{r 0}$ as a function of bias voltage. The maximum $f_{r 0}$ of $5.6 \mathrm{GHz}$ was obtained at a $580-\mathrm{mV}$ bias voltage.

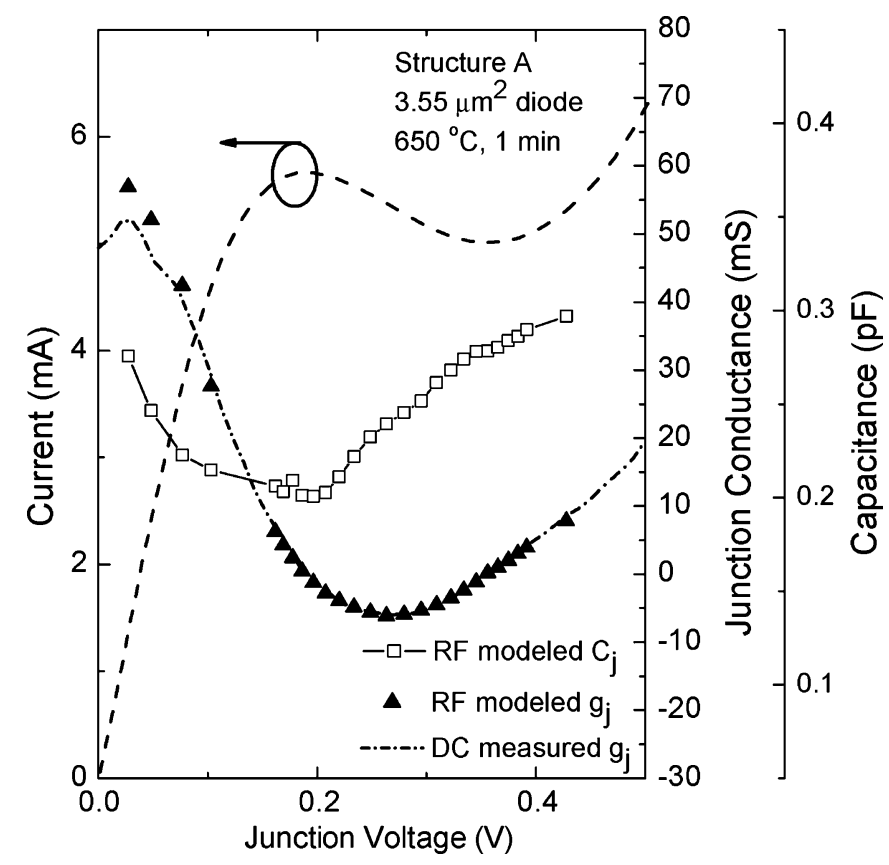

Fig. 5. Extracted $g_{j}$ and $C_{j}$ are plotted as a function of junction voltage for Structure A annealed at $650{ }^{\circ} \mathrm{C}$. The intrinsic dc $I-V$ characteristics are superimposed. $g_{j}$ is also computed from the intrinsic dc $I-V$ characteristic. Very good agreement between RF modeled and dc measured $g_{j}$ is obtained.

good agreement with the measured values. The bias independent elements $r_{s}$ and $L_{s}$ were extracted as $60.7 \Omega$ and 0.41 $\mathrm{nH}$, respectively. Fig. 5 shows the extracted junction capacitance, $C_{j}$, as a function of the actual junction voltage, which 


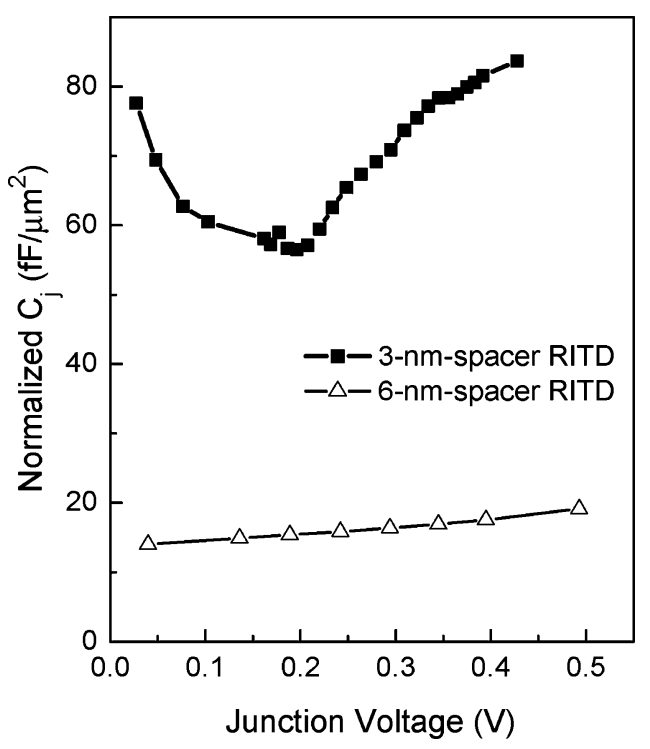

Fig. 6. Comparison of normalized $C_{j}$ of Structure A annealed at $650{ }^{\circ} \mathrm{C}$ and Structure B annealed at $825^{\circ} \mathrm{C}$.

was computed by subtracting the voltage drop across $r_{s}$ from the total external bias voltage. The dc $I-V$ characteristic of the intrinsic tunnel diode (deembedded from $r_{s}$ ) is then superimposed in Fig. 5 as the dashed line. The extracted junction conductance, $g_{j}$, as a function of junction voltage is also shown in Fig. 5. The $g_{j}$ was also computed by taking the first derivative of the intrinsic de $I-V$ characteristic. The computed $g_{j}$ was then plotted in Fig. 6 as the dashed-dotted line. Excellent agreement between the junction conductance obtained from RF measurements and dc measurements was observed, which indicates the equivalent circuit model, shown in Fig. 3, fully describes the physical processes [14]. The excellent agreement also suggests that bias dependent $g_{j}$ can be accurately computed from the intrinsic de $I-V$ characteristic without taking RF measurements. When the bias voltage is $580 \mathrm{mV}$ in the NDR region (corresponding to a junction voltage of $249 \mathrm{mV}$ ), the extracted $C_{j}$ and $g_{j}$ are $232 \mathrm{fF}$ and $-5.6 \mathrm{mS}$, respectively. According to (3) [15], $f_{r 0}$ is calculated to be $5.4 \mathrm{GHz}$, which is in close agreement to the measured value of $5.6 \mathrm{GHz}$

$$
f_{r 0}=\frac{\left|g_{j}\right|}{2 \pi C_{j}} \sqrt{\frac{1}{r_{s}\left|g_{j}\right|}-1}
$$

The speed index can be calculated as $s=\mathrm{Ip} / \mathrm{C}=$ $5.4 \mathrm{~mA} / 232 \mathrm{fF}=23.3 \mathrm{mV} / \mathrm{ps}$, which is also the highest value ever reported for any epitaxially grown Si-based tunnel diode. Table I summarizes the prior measured $f_{r 0}$ and speed index of various $\mathrm{Si}$-based tunnel diodes reported by different groups.

The maximum $f_{r 0}$ of Structure B annealed at $825^{\circ} \mathrm{C}$ was determined to be $0.4 \mathrm{GHz}$ at a junction voltage of $196 \mathrm{mV}$ for a $10 \mu \mathrm{m}$ diameter device. The same $S_{11}$ parameter fitting procedure was performed to extract its circuit element values. To understand the performance difference between the two RITDs, the extracted $C_{j},\left|g_{j}\right|$, and $r_{s}$ of both Structure A and Structure $\mathrm{B}$ at their best bias voltages were normalized with junction area and summarized in Table II with other important parameters.
TABLE I

PREVIOUSLY REPORTED PERFORMANCE OF VARIOUS EPITAXIALLY GROWN Si-BAsed Tunnel Diodes By Different Groups. Note, ONLY TunNEL Diodes With MEASURED $f_{r 0}$ AND SPEED INDEX ARE Listed

\begin{tabular}{ccccccc}
\hline First Author/year & Type & $\begin{array}{c}\text { Fabrication } \\
\text { Method }\end{array}$ & $\begin{array}{c}f_{r 0} \\
(\mathrm{GHz})\end{array}$ & $\begin{array}{c}\text { Speed index } \\
(\mathrm{mV} / \mathrm{ps})\end{array}$ & $\begin{array}{c}\text { PCD } \\
\left(\mathrm{kA} / \mathrm{cm}^{2}\right)\end{array}$ & PVCR \\
\hline Auer [11], 2001 & Si/SiGe ITD & MBE & 1.5 & 0.45 & 1.52 & 2.1 \\
Dashiell [12], 2002 & Si Esaki & MBE & 1.6 & 7.1 & 16 & 1.04 \\
Yan [16], 2004 & Si Esaki & RTP* & $\sim 0.45$ & 0.23 & 0.27 & 2.15 \\
This work & Si/SiGe RITD & MBE & 5.6 & 24.6 & 152 & 1.14 \\
\hline
\end{tabular}

* Rapid thermal processing

Assuming $r_{s} \cdot\left|g_{j}\right| \ll 1$, the ratio of the maximum $f_{r 0}$ of two tunnel diodes can be approximated as

$$
\frac{f_{r 0}^{A}}{f_{r 0}^{B}}=\frac{\sqrt{\left|g_{j}^{A}\right| \cdot r_{s}^{B}} \cdot C_{j}^{B}}{\sqrt{\left|g_{j}^{B}\right| \cdot r_{s}^{A}} \cdot C_{j}^{A}} .
$$

With a smaller spacer thickness and lower annealing temperature, Structure A has a thinner tunneling barrier than Structure B. Also, the Ge content within the spacer layer of Structure A is higher than that of Structure B, which lowers the tunneling barrier height, increases momentum mixing, and consequently further increases the tunneling probability. These factors lead to a $\sim 4.2$ times larger normalized $C_{j}$ and $\sim 155$ times higher normalized $\left|g_{j}\right|$, as shown in Table II. According to (4), this results in a $\sim 3.0$ times improvement of $f_{r 0}$. Table II also shows that the normalized $r_{s}$ of Structure A is significantly smaller than Structure B, which leads to a $\sim 4.7$ times improvement of $f_{r 0}$ according to (4). There are two reasons which account for the significant difference in normalized $r_{s}$ : First, the Ni silicide ohmic contact is only used to reduce the series resistance for Structure A. Second, the contact resistance does not scale accordingly with area [17], i.e., Structure A will have a smaller normalized $r_{s}$ even if the same Ni silicide ohmic contact is used in both diodes.

Equation (4) and the comparison between Structures A and B suggest several approaches to increase $f_{r 0}$ of Si-based tunnel diodes:

1) An ultrathin tunneling barrier is desirable for high $f_{r 0}$, even though it will lead to a larger normalized $C_{j}$. This is because the PCD exponentially increases as the tunnel barrier thickness decreases, causing an exponential increase of normalized $\left|g_{j}\right|$ (even with a unfavorable PVCR drop [5]), which will outweigh the losses from the linearly increasing normalized $C_{j}$.

2) Increase the Ge content within spacer layer to enhance the tunneling probability, hence normalized $\left|g_{j}\right|$, with only a slightly increased normalized $C_{j}$ due to higher dielectric constant of Ge.

3) Reduce contact resistance thereby normalized $r_{s}$. Extremely low contact resistance in the range of $10^{-7} \Omega \cdot \mathrm{cm}^{2}$ has already been obtained from state-of-the-art Ni silicide technology [18], [19]. The implementation of such $\mathrm{Ni}$ silicidation process on Structure A would result in a normalized $r_{s}$ in the range of $10 \Omega \cdot \mu \mathrm{m}^{2}$, which could lead to a $f_{r 0}$ of $26.6 \mathrm{GHz}$ (4.7 times improvement). 
TABLE II

COMPARISON BETWEEN RITDS OF STRUCTURE A AND B. THE NORMALIZED $C_{j},\left|g_{j}\right|$ OF STRUCTURE A AND B ARE the VAlues at JUNCTION VOLTAGE OF 249 AND $196 \mathrm{mV}$, RESPECTIVELY

\begin{tabular}{lll}
\hline & Structure A & Structure B \\
\hline Spacer thickness $(\mathrm{nm})$ & 3 & 6 \\
Annealing temp $\left({ }^{\circ} \mathrm{C}\right)$ & 650 & 825 \\
Ge content & $50 \%$ & $40 \%$ \\
Diode area $\left(\mu \mathrm{m}^{2}\right)$ & 3.55 & 78.50 \\
PVCR & 1.14 & 2.9 \\
PCD $\left(\mathrm{kA} / \mathrm{cm}^{2}\right)$ & 152 & 0.316 \\
Speed index $(\mathrm{mV} / \mathrm{ps})$ & 23.3 & 0.2 \\
Maximum $\boldsymbol{f}_{\boldsymbol{r} \boldsymbol{0}}(\mathrm{GHz})$ & 5.6 & 0.4 \\
Normalized $\left|\boldsymbol{g}_{j}\right|\left(\mathrm{ms} / \mu \mathrm{m}^{2}\right)$ & 1.55 & 0.01 \\
Normalized $\boldsymbol{C}_{\boldsymbol{j}}\left(\mathrm{fF} / \mu \mathrm{m}^{2}\right)$ & 65.3 & 15.4 \\
Normalized $\boldsymbol{r}_{\boldsymbol{s}}\left(\Omega \cdot \mu \mathrm{m}^{2}\right)$ & 216 & 4789 \\
\hline
\end{tabular}

4) Scaling down RITD will reduce its normalized $r_{s}$, thereby improve $f_{r 0}$, assuming the normalized $C_{j}$ and $\left|g_{j}\right|$ are area independent.

It is interesting to note for Structure A that $C_{j}$ reaches its minimum value when the current reaches its peak value (Fig. 5). The observed capacitance-voltage $(C-V)$ trend is significantly different from the $C-V$ trend reported for an Esaki diode [20], in which the Esaki junction capacitance monotonically increases with the bias voltage as a result of a narrowed depletion region. The anomalous capacitance change for these Si-based RITDs may result from charge redistribution within the triangular quantum well as the bias voltage is varied. The $825^{\circ} \mathrm{C}$ annealed Structure B shows a monotonically increased $C_{j}$ with junction voltage (Fig. 6), which is characteristic of Esaki diodes. The different $C-V$ relationship of $650{ }^{\circ} \mathrm{C}$ annealed and $825^{\circ} \mathrm{C}$ annealed RITDs may suggest that annealing at too high a temperature $\left(825^{\circ} \mathrm{C}\right)$ will diffuse dopants enough that the $\delta$-doped quantum wells may lose their confinement, effectively rendering the RITD structure into an Esaki diode.

\section{COMPREHENSIVE DC/RF MODEL}

A comprehensive dc/RF model which incorporates both the equivalent circuit model and dc $I-V$ characteristics was developed for InGaAs/InAlAs ITDs in monolithic microwave integrated circuits [9]. In this section, a similar dc/RF model for Structure B is developed.

Fig. 7 shows the comprehensive dc/RF ITD model. $R_{s}$ is the series resistance determined in the small signal model. $C_{j}(V)$ is a fitted polynominal function of the extracted $C_{j}$. The $I_{j}(V)$ is the intrinsic de $I-V$ characteristics which is deembedded from the $R_{s}$ effect. In the InGaAs/InAlAs ITDs dc/RF model [9], $I_{j}(V)$ is a polynominal fit of the measured data, which is not

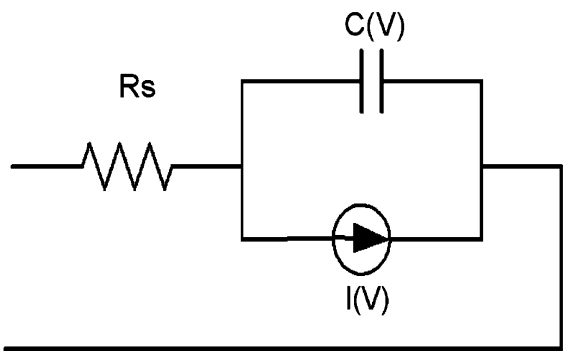

Fig. 7. Comprehensive dc/RF model of the RITDs studied here.

TABLE III

PARAMETER VAlues After FitTing the $I-V$ Characteristics FOR STRUCTURE B ANNEALED AT $825^{\circ} \mathrm{C}$

\begin{tabular}{ll}
\hline Parameter & Value \\
\hline$I_{p}$ & $2.4 \times 10^{-4} \mathrm{~A}$ \\
$V_{p}$ & $0.01 \mathrm{~V}$ \\
$I_{e}$ & $1.7 \times 10^{-6} \mathrm{~A}$ \\
$W$ & 8.0 \\
$I_{0}$ & $3.0 \times 10^{-13} \mathrm{~A}$ \\
$n$ & 1.3 \\
\hline
\end{tabular}

physically meaningful. In our semi-physical model, the forward $I-V$ characteristics of the RITD was modeled by the summation of its three current components: tunneling current $I_{t}$, thermal diffusion current $I_{\mathrm{th}}$, and excess current $I_{e}$.

The tunneling current can be expressed using the empirical equation [21]

$$
I_{t}=I_{p} \times\left(\frac{V}{V_{p}}\right) \times \exp \left(1-\frac{V}{V_{p}}\right)
$$

where $I_{p}$ is the peak tunneling current, $V_{p}$ is the peak voltage, and $V$ is the bias voltage. The thermal diffusion current is the standard diode equation expressed as

$$
I_{\mathrm{th}}=I_{0} \times \exp \left(\frac{V}{n k T}-1\right)
$$

where $I_{0}$ is the reverse saturation current, $n$ is the ideality factor, and $k T$ is the thermal energy. Since the excess current is exponentially dependent on the bias voltage as suggested by Chynoweth [22], it can be simply expressed as

$$
I_{e}=I_{e} \times \exp (W \times V)
$$

where $I_{e}$ and $W$ are constant.

The parameters $I_{p}, V_{p}, I_{e}, W, I_{0}$, and $n$ were optimized to fit the intrinsic $I-V$ characteristics for Structure B using ADS software. The parameter values resulting from the optimization are listed in Table III. Fig. 8 shows the intrinsic $I-V$ characteristics and the modeled $I-V$ characteristics, as well as the three current components. Excellent agreement between the intrinsic and modeled $I-V$ characteristics was obtained.

The comprehensive dc/RF model shown in Fig. 7 was then implemented using ADS software to simulate the instability and oscillation that are commonly observed during dc electrical measurements. The tungsten wire probe commonly used for $\mathrm{dc}$ measurements acts as a series inductor, which causes oscillations during dc measurements when the second stable condition (Inequality 2) is no longer met. The commonly observed 


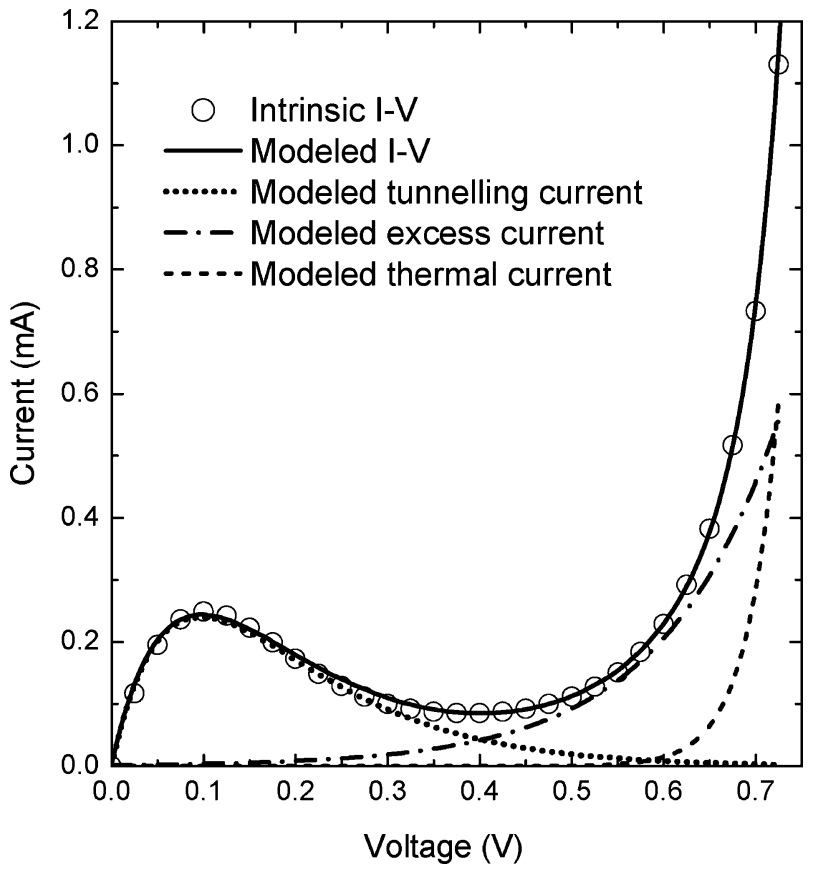

Fig. 8. Comparison of intrinsic and modeled $I-V$ characteristic. The three modeled current components band-to-band tunneling, diffusion, and excess current are also plotted.

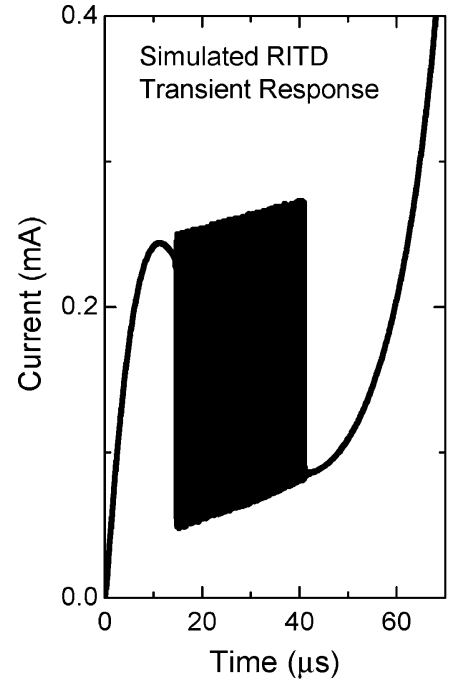

(a)

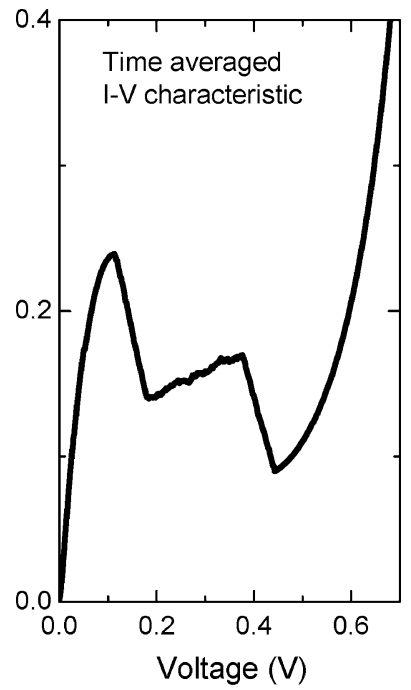

(b)
Fig. 9. (a) Simulated transient current showing strong oscillations when Structure B annealed at $825^{\circ} \mathrm{C}$ is biased in the NDR region. (b) Simulated time-averaged $I-V$ characteristic showing the plateau in the NDR region.

plateau, or hump in the NDR region is an indication of these oscillations and sometimes appears singly or multiply. A transient simulation was performed to emulate the response of the measured diode with a serially connected $50 \mu \mathrm{H}$ inductor to a bias voltage ramped from 0 to $1 \mathrm{~V}$ in $0.1 \mathrm{~ms}$. The simulated transient current was then averaged over time to mimic the integration process of the dc measurement using a parameter analyzer. Fig. 9(a) shows the simulated transient current, where strong oscillations occur when the diode is biased into its NDR region. Fig. 9(b) shows the resulting time-averaged $I-V$ characteristic, a plateau in the NDR region was observed.

\section{CONCLUSION}

The RF performance of two different Si-based RITD structures were studied. Structure A annealed at $6500^{\circ} \mathrm{C}$ exhibits an $f_{r 0}$ of $5.6 \mathrm{GHz}$ and a speed index of $23.3 \mathrm{mV} / \mathrm{ps}$, which are both the highest values ever reported for any epitaxially grown Si-based tunnel diodes. Structure B annealed at $825{ }^{\circ} \mathrm{C}$ shows an $f_{r 0}$ of $0.4 \mathrm{GHz}$. The performance difference between these two diodes suggests thinner tunneling barrier, higher Ge content within spacer, better ohmic contact, and smaller junction area are desirable for higher $f_{r 0}$. A $f_{r 0}$ well over $10 \mathrm{GHz}$ can be obtained by optimizing the growth and fabrication process. A comprehensive de/RF model was developed and implemented in ADS. The instabilities in the NDR region during dc measurements were simulated.

\section{ACKNOWLEDGMENT}

The authors would like to thank D. Liu for her assistance in RF measurements, Prof. W. Lu for technical discussions on parameter extraction, Prof. P. Roblin for technical discussions about instability during de measurements, and Dr. W. Dai for technical discussions about modeling in ADS.

\section{REFERENCES}

[1] S. L. Rommel, T. E. Dillon, M. W. Dashiell, H. Feng, J. Kolodzey, P. R. Berger, P. E. Thompson, K. D. Hobart, R. Lake, A. C. Seabaugh, G. Klimeck, and D. K. Blanks, "Room temperature operation of epitaxially grown $\mathrm{Si} / \mathrm{Si}_{0.5} \mathrm{Ge}_{0.5} / \mathrm{Si}$ resonant interband tunneling diodes," Appl. Phys. Lett., vol. 73, pp. 2191-2193, 1998.

[2] P. E. Thompson, K. D. Hobart, M. Twigg, G. Jernigan, T. E. Dillon, S. L. Rommel, P. R. Berger, D. S. Simons, R. Lake, and A. C. Seabaugh, "Si resonant interband tunnel diodes grown by low temperature molecular beam epitaxy," Appl. Phys. Lett., vol. 75, pp. 1308-1310, 1999.

[3] K. Eberl, R. Duschl, O. G. Schmidt, U. Denker, and R. Haug, "Si-based resonant inter- and intraband tunneling diodes," J. Cryst. Growth, vol. 227-228, pp. 770-776, 2001.

[4] N. Jin, S.-Y. Chung, A. T. Rice, P. R. Berger, P. E. Thompson, C. Rivas, R. Lake, S. Sudirgo, J. J. Kempisty, B. Curanovic, S. L. Rommel, K. D. Hirschman, S. K. Kurinec, P. H. Chi, and D. S. Simons, "Diffusion barrier cladding in $\mathrm{Si} / \mathrm{SiGe}$ resonant interband tunneling diodes and their patterned growth on pMOS source/drain regions," IEEE Trans. Electron Devices, vol. 50, no. 11, pp. 1876-1884, Nov. 2003.

[5] N. Jin, S.-Y. Chung, A. T. Rice, P. R. Berger, R. Yu, P. E. Thompson, and R. Lake, " $151 \mathrm{kA} / \mathrm{cm}^{2}$ peak current densities in $\mathrm{Si} / \mathrm{SiGe}$ resonant interband tunneling diodes for high-power mixed-signal applications," Appl. Phys. Lett., vol. 83, pp. 3308-3310, 2003.

[6] N. Jin, S.-Y. Chung, R. M. Heyns, P. R. Berger, R. Yu, P. E. Thompson, and S. L. Rommel, "Tri-state logic using vertically integrated Si resonant interband tunneling diodes with double NDR," IEEE Electron Device Lett., vol. 25, no. 6, pp. 646-648, Jun. 2004.

[7] S. Y. Chung, N. Jin, P. R. Berger, R. Yu, P. E. Thompson, R. Lake, S. L. Rommel, and S. K. Kurinec, "3-terminal Si-based negative differential resistance circuit element with adjustable peak-to-valley current ratios using a monolithic vertical integration," Appl. Phys. Lett., vol. 84, pp. 2688-2690, 2004.

[8] S. Sudirgo, R. P. Nandgaonkar, B. Curanovic, J. L. Hebding, R. L. Saxer, S. S. Islam, K. D. Hirschman, S. L. Rommel, S. K. Kurinec, P. E. Thompson, N. Jin, and P. R. Berger, "Monolithically integrated Si/SiGe resonant interband tunnel diode/CMOS demonstrating low voltage MOBILE operation," Solid State Electron., vol. 48, pp. 1907-1910, 2004.

[9] A. Cidronali, V. Nair, G. Collodi, J. H. Lewis, M. Camprini, G. Manes, and H. Goronkin, "MMIC applications of heterostructure interband tunnel devices," IEEE Trans. Microw. Theory Tech., vol. 51, no. 11, pp. 1351-1367, Nov. 2003.

[10] A. Seabaugh and R. Lake, "Tunnel diodes," in Encyclopedia of Applied Physics. New York: Wiley, 1998, vol. 22, pp. 335-359.

[11] U. Auer, W. Prost, M. Agethen, F. J. Tegude, R. Duschl, and K. Eberl, "Low-voltage MOBILE logic module based on $\mathrm{Si} / \mathrm{SiGe}$ interband tunnelling diodes," IEEE Electron Device Lett., vol. 22, no. 5, pp. 215-217, May 2001. 
[12] M. W. Dashiell, J. Kolodzey, P. Crozat, F. Aniel, and J. M. Lourtioz, "Microwave properties of silicon junction tunnel diodes grown by molecular beam epitaxy," IEEE Electron Device Lett., vol. 23, no. 6, pp. 357-359, Jun. 2002

[13] C. Y. Belhadj, K. P. Martin, S. B. Amor, J. J. L. Rascol, R. J. Higgins, R. C. Potter, H. Hier, and E. Hempfling, "Bias circuit effects on the current-voltage characteristic of double-barrier tunneling structures: experimental and theoretical results," Appl. Phys. Lett., vol. 57, pp. 58-60, 1990.

[14] P. Fay, J. Lu, Y. Xu, N. Dame, D. H. Chow, and J. N. Schulman, "Microwave performance and modeling of InAs/AlSb/GaSb resonant interband tunneling diodes," IEEE Trans. Electron Devices, vol. 49, no. 1, pp. 19-24, Jan. 2002.

[15] C. Kidner, I. Mehdi, J. R. East, and G. I. Haddad, "Power and stability limitations of resonant tunneling diodes," IEEE Trans. Microw. Theory Tech., vol. 38, no. 8, pp. 864-872, Aug. 1990.

[16] Y. Yan, J. Zhao, Q. Liu, W. Zhao, and A. Seabaugh, "Vertical tunnel diodes on high resistivity silicon," in Proc. 62nd Device Research Conf., 2004, pp. 27-28.

[17] S. Cohen, "Contact resistance and methods for its determination," Thin Solid Films, vol. 104, pp. 361-379, 1983.

[18] J. Eberhardt and E. Kasper, "Ni/Ag metallization for SiGe HBTs using a Ni silicide contact," Semicond. Sci. Technol., vol. 16, pp. L47-L49, 2001

[19] M. C. Ozturk, J. Liu, H. Mo, and N. Pesovic, "Advanced SiGe source/drain and contact technologies for sub-70 nm CMOS," in IEDM Tech. Dig., 2002, pp. 375-378.

[20] H. Fukui, "The characteristics of Esaki diodes at microwave frequencies," in Proc. IEEE Int. Solid-State Circuits Conf., vol. IV, 1961, pp. $16-17$.

[21] T. A. Demassa and D. P. Knott, "The prediction of tunnel diode voltage-current characteristics," Solid State Electron., vol. 13, pp. $131-138,1970$

[22] A. G. Chynoweth, W. L. Feldmann, and R. A. Logan, "Excess tunnel current in silicon Esaki junctions," Phys. Rev., vol. 121, pp. 684-694, 1961.

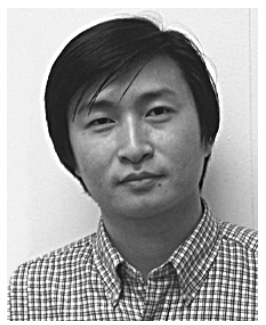

Niu Jin (S'03-M'04) received the B.E. and M.E. degrees in materials science and engineering from Hefei University of Technology, Hefei, China, in 1995 and 1998, respectively, and the M.S. and Ph.D. degree in electrical engineering at The Ohio State University, Columbus, in 2001 and 2004, respectively, where he worked on Si-based tunneling devices.

Currently, he is a Postdoctoral Research Associate at the University of Illinois, Urbana-Champaign, where he works on nanofluidic devices.

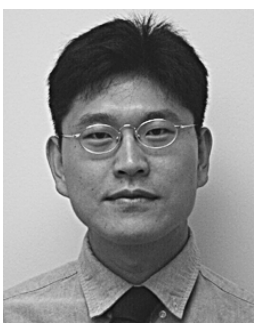

Sung-Yong Chung (S'00) received the B.S. degree in metallurgical engineering from Korea University, Seoul, Korea, in 1999, and the M.S. degree in electrical engineering from The Ohio State University, Columbus, in 2002, where he is currently pursuing the Ph.D. degree.

His current research interests include the design, fabrication, and characterization of SiGe-based nanoelectronics, and SiGe-based microwave device and circuits.

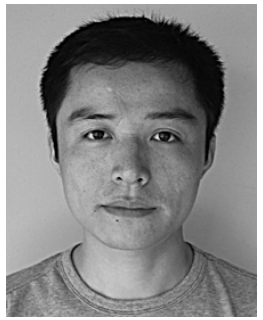

Ronghua Yu received the B.S. degree from the University of Electronic Science and Technology of China, Sichuan, China, in 1994, and the M.S. from the University of Science and Technology of China, Anhui, China, in 1997, both in physics. $\mathrm{He}$ is currently pursuing the Ph.D. degree at The Ohio State University, Columbus.

His research interests include semiconductor physics, and the physics and technology of nanometer-scaled materials and structures. His current research activities are centered around $\mathrm{Si} / \mathrm{SiGe}$-based resonant interband tunnel diodes.

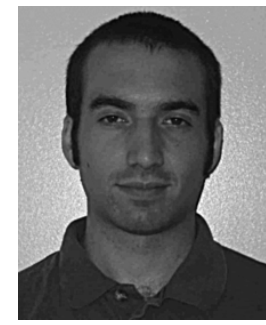

Sandro J. Di Giacomo (S'98-M'05) received the B.S. degree in electrical engineering degree (cum laude) from the University of Delaware, in 2002 and the M.S. degree in electrical and computer engineering from The Ohio State University, Columbus, in 2005 .

Currently, he is a Process Engineer at the Northrop Grumman Advanced Materials and Semiconductor Device Technology Center, Baltimore, MD, where he works on Si-based CVD and VPE process development and SONOS device development.

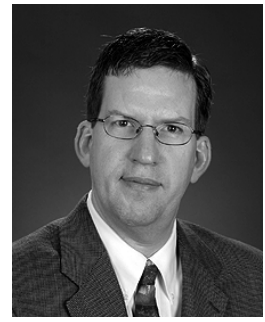

Paul R. Berger (S'84-M'91-SM'97) received the B.S.E. degree in engineering physics in 1985 , and the M.S.E. and Ph.D. degrees in electrical engineering in 1987 and 1990, respectively, all from the University of Michigan, Ann Arbor.

$\mathrm{He}$ is currently a Professor in both the Electrical and Computer Engineering Department and the Physics Department at The Ohio State University. $\mathrm{He}$ is the Founder and Director of Ohio's new Nanoscale Patterning Center. Currently, he is actively working on $\mathrm{Si} / \mathrm{SiGe}$ nanoelectronic devices and fabrication processes, Si-based resonant interband tunneling diodes and quantum functional circuitry, conjugated polymer-based optoelectronic and electronic devices, and semiconductor materials, fabrication and growth. From 1985 to 1990, he worked on III-V compound MBE, InGaAs/GaAs strained layer growth and self-assembled quantum dots, and optoelectronic devices and integrated circuits. From 1990 to 1992 he worked at Bell Laboratories, Murray Hill, NJ on optoelectronic devices and OEICs. He was an Assistant Professor from 1992 to 1997 and an Associate Professor from 1997 to 2000 at the University of Delaware, Newark, in electrical and computer engineering. In 1999, a sabbatical leave lead him to work at the Max-Planck Institute for Polymer Research, Mainz, Germany and Cambridge Display Technology, Ltd. Cambridge, U.K. In 2000, Dr. Berger joined The Ohio State University as an Associate Professor. He has coauthored over 70 archival refereed journal articles, over 70 invited and regular conference presentations, three book sections and been issued six U.S. patents with seven more pending.

Dr. Berger is a recipient of the National Science Foundations 1996 Faculty Early Career Development Award. In 1998, he received the DARPA ULTRA Sustained Excellence Award. He was a co-organizer of the SRC-sponsored Si Tunnel Diode and CMOS/HBT Integration Workshop in Washington, DC. He has been on the Program and Advisory Committees of the International Electron Devices Meeting, International Semiconductor Device Research Symposium, Electronic and Photonic Materials, Devices and Systems, and SPIE conferences. $\mathrm{He}$ is a member of the Optical Society of America and the Materials Research Society.

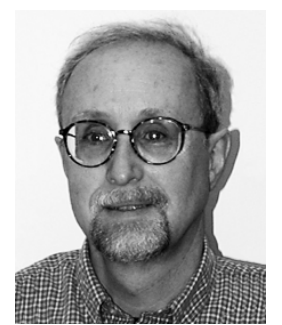

has three pending.

Dr. Thompson was a co-organizer of the SRC-sponsored Si Tunnel Diode and CMOS/HBT Integration Workshop in Washington, DC. He received two NRL Tech Transfer Awards and the DARPA ULTRA Sustained Excellence Award in 1998. He is a member of APS.
Phillip E. Thompson (SM'00) received the Ph.D. degree in physics from the University of Delaware, Newark, in 1975.

$\mathrm{He}$ is the Head of the Si MBE Growth and Characterization Section, Code 6812, Electronics Science and Technology Division, Naval Research Laboratories (NRL), Washington DC. He joined NRL in 1981 as a Research Physicist. He has over 22 years experience in electronic materials, including 15 years in MBE growth. He has over 190 publications and presentations. He holds five patents and 